\title{
Social feeding in domestic chicks: A test of the disinhibition hypothesis'
}

\author{
C. W. TOLMAN AND A. W. WELLMAN, DEPARTMENT OF \\ PSYCHOLOGY, UNIVERSITY OF VICTORIA, Victoria, B. C., \\ Canada
}

The hypothesis tested was that social facilitation of feeding among young domestic chicks could be accounted for by the disinhibitory effects of a companion's presence. The hypothesis predicted that pre-exposure to the test environment will decrease the apparent facilitative effect of the companion. Sixty chicks were tested either as singles or pairs $24 \mathrm{~h}$ following either no exposure, exposure as singles or exposure as pairs. Exposure increased feeding but independently of differences between singles and pairs, thus disconfirming the hypothesis.

According to Crawford (1939) social facilitation refers to "any increment of individual activity which results from the presence of another individual." A recent review of the social facilitation literature (Tolman, 1968) revealed that at least four distinct hypotheses have been advanced by various authors to account for this phenomenon. The "reflex hypothesis" states that some aspect of the companion, as well as the apparent nonsocial stimulus, elicits the response from $S$, thus causing the increment. The "motivational hypothesis" states that the companion causes an increase in S's overall arousal level. The "perception hypothesis" states that the companion elicits attending responses to the nonsocial stimulus, which then elicits the measured response. According to the "disinhibition hypothesis," $S$ is inhibited from performing the response, and the companion causes a reduction of this inhibition.

Regarding the social facilitation of feeding behavior in domestic chicks, all of these hypotheses have been entertained at one time or another. Most serious attention, however, has been restricted to the reflex and disinhibition hypotheses (Tolman, 1968). While the disinhibition hypothesis has been much discussed, the evidence, both for and against it, has been weak and indirect (Tolman, 1965 Tolman \& Wilson, 1965).

The present experiment attempts to test the disinhibition hypothesis. A socially reared chick placed alone in a strange environment exhibits behavior from which has been inferred a state of "fear," "distress," and even "loneliness" (Grindley, 1929). Such behavior, or its inferred state, might inhibit feeding in a moderately hungry bird. The disinhibition hypothesis would argue that the presence or activity of a companion reduces this emotionality, thus disinhibiting feeding. It would also predict that any other operation reducing the emotionality would cause an increase in S's feeding. Since the functions of the companion and the other disinhibiting operation are equivalent, the apparent effect of the companion should be reduced or eliminated by the prior introduction of the other operation. This expectation is suggested by the finding that two or more companions produce no greater facilitative effect than one (Tolman \& Wilson, 1965). Presuming the effect to be disinhibitory, it would have to be concluded that one companion is maximally disinhibiting. The addition of a nonsocial source of disinhibition, then, would reduce the apparent effect of the companion.

Assuming that a large share of the single chick's distress is accounted for by the strangeness of the test environment, prior exposure to that environment was used in the present experiment as the nonsocial disinhibiting operation. On the first day of the experiment birds were exposed as pairs of singles. A third group was not exposed. On the second day each group was subdivided and tested as singles or pairs. The disinhibition hypothesis predicts (1) that exposed birds will eat more than nonexposed birds (this also provided a test of the effectiveness of the exposure as a disinhibiting operation) and (2) that there will be an interaction between exposure and test condition such that the difference between singles and pairs should be less for the exposed groups than for the nonexposed group.

Subjects. Sixty white Leghorn cockerels obtained as day-olds from a local commercial hatchery were tested when four-five days of age. Equal numbers of Ss were maintained in each of four identical brooder compartments. Water and food, a locally prepared chick starter mash, were continuously available in the brooder with the exception of the periods of food deprivation described below. A $6 \mathrm{~W}$ incandescent lamp in each brooder compartment provided constant illumination.

Apparatus. The test apparatus was a rectangular observation box with clear plastic walls, $10 \times 18 \times 10$ in. It was divided into two compartments of equal size by the insertion of a 3/32 in. masonite partition into vertical grooves in the side walls. The wood floor and the partition were finished in a gray enamel paint. The floor of the observation box was spread evenly with chick starter mash, forming a layer approximately $1 / 8$ in. deep. Two $100 \mathrm{~W}$ desk lamps were placed between the Os and the test box, illuminating the observation areas from central points 12 in. above the floor of each of the two compartments. The temperature in the observation boxes was the same as that in the brooder, about $88^{\circ} \mathrm{F}$.

Procedure. The experiment took place between 12:00 N and 4:30 PM on two consecutive days. All Ss underwent $4-6 \mathrm{~h}$ food deprivation prior to being placed in the test apparatus on both of these days. Half the Ss were deprived at 8:00 AM and the other half at 10:00 PM. Water was continuously available in the brooder but was not provided in the test apparatus.

On the first day, two groups of 20 Ss each were put into the test apparatus for 7-min exposure sessions. In one group, pairs of Ss were placed into one compartment of the observation box. In the other group, single Ss were placed into each of the compartments of the observation box. A third group of $20 \mathrm{Ss}$ was not given exposure sessions in the test apparatus. Food was present in the observation box.

On the second day, each of the three groups was divided into two subgroups of 10 birds, one being tested as pairs and the other as singles. The test sessions were 7 min long.

The response recorded for each $S$ was the total number of food directed pecks emitted by $S$ during the test session.

Results and Discussion. The resulting means are presented in Table 1. An analysis of variance indicated a significant effect of test conditions, paired and single feeding $(F=7.21$, df $=2 / 54, p<$ $.01)$, a significant effect of exposure $(F=14.61$, df $=2 / 54, p<$ $.01)$, but no significant interaction $(F=1.85$, df $=2 / 54)$. A partitioning of the exposure effects showed that the significant variation was due to exposure vs nonexposure $(\mathrm{F}=28.59, \mathrm{df}=$ $1 / 54, p<.01)$, not to single vs paired exposure $(F=0.63$, df $=$ $1 / 54)$.

The first prediction of the disinhibition hypothesis, that exposure to the test environment would cause, through reduction of interfering responses or emotional condition, an increase in feeding was unequivocally confirmed. This prediction, however, was in no way crucial to the viability of the hypothesis. Had it not been confirmed, it would simply have been regarded as a failure to select an operation that met the requirements of the hypothesis. Confirmation in this case, however, demonstrates that the exposure was adequate and thus permits an examination of the second prediction.

The prediction that there would be an interaction between

Table 1

Mean Pecks Emitted During Test, Day 2

\begin{tabular}{cccc} 
Test Condition & \multicolumn{3}{c}{ Day 1 Exposure Condition } \\
\cline { 2 - 4 } Day 2 & No Exposure & Single Chicks & Paired Chicks \\
\hline Paired & 60.7 & 423.8 & 408.3 \\
Single & 41.1 & 177.3 & 286.5 \\
\hline
\end{tabular}


exposure and testing conditions provided a crucial test of the disinhibition hypothesis as far as social facilitation is concerned. As can readily be seen from the statistical results, this expectation was not met. While prior exposure to the test situation clearly increased feeding, it did so independently of the social increase in feeding.

The adherent of the disinhibition hypothesis has yet one recourse. He could argue that an analysis of variance evaluates only absolute differences between groups: the social facilitation effect should be viewed as a percentage increase. The present data, however, do not yield to this shift of focus. The percentage increases were $47.7,71.9$, and 42.5 for the no-exposure, single exposure, and paired exposure groups respectively. There is no suggestion here that the social effect was decreased by prior exposure.

The fact that the effect of pretest exposure to the test situation was independent of whether the chick had a partner or was alone during exposure may be taken as further evidence for the lack of similarity between disinhibitory and social effect. ${ }^{2}$

It may be concluded that the feeding behavior of chicks in a novel test environment can be considerably increased by the disinhibitory effect of pretest exposure, but this does not alter the social effect which appears not to be of a disinhibitory nature..

\section{REFERENCES}

CRAWFORD, M. P. The social psychology of the vertebrates. Psychol. Bull., $1939,36,407-446$.

GRINDLEY, G. C. Experiments on the influence of the amount of reward on learning in young chickens. Brit. J. Psychol., 1929, 20, 173-180.

TOLMAN, C. W. Emotional behavior and social facilitation of feeding in domestic chicks. Anim Behav., 1965, 13, 493-496.

TOLMAN, $\mathrm{C}$. W. The role of the companion in social facilitation of animal behavior. In E. Simmel, R. Hoppe, \& G. Milton (Eds.), Social facilitation and imitative behavior. Boston: Allyn and Bacon, 1968.

TOLMAN, C. W., \& WLSON, G. F. Social feeding in domestic chicks. Anim. Behav., 1965, 13, 134-142.

\section{NOTES}

1. This work was supported by research grant APA 245 from the National Research Council of Canada.

2. The apparent interaction in the paired and single exposure columns was separately analyzed and found to be not significant $(F=1.14)$. 